\title{
Sismicidad y percepción del riesgo en el departamento de Ica, Perú
}

Seismicity and risk perception in the departament of Ica, Peru

Recibido: setiembre 01 de 2021 | Revisado: octubre 02 de 2021 | Aceptado: noviembre 10 de 2021

\author{
Pedro Félix Doroteo Neyra ${ }^{\mathrm{I}}$ \\ Martín Hamilton Wilson Huamanchumo ${ }^{\mathrm{I}}$ \\ George Argota Pérez ${ }^{2}$
}

\section{Resumen}

Los sismos son eventos adversos naturales que generan pérdidas humanas y daños materiales. El objetivo del estudio fue analizar la sismicidad y percepción del riesgo en el departamento de Ica, Perú. Se seleccionó la ciudad de Pisco por el antecedente sismológico que se registró el 15 de agosto de 2007. Mediante un muestreo probabilístico aleatorio, 10 viviendas a $1 \mathrm{Km}$ de distancia entre ellas y cercanas a la línea de costa fueron seleccionadas para el análisis de vulnerabilidad. Se aplicó una encuesta a un miembro del núcleo familiar y que correspondió a cuatro de las 10 viviendas y analizó la manifestación poblacional residente ante el riesgo por sismos y la vulnerabilidad de las viviendas. La escala Likert indicó que el conocimiento de la población según el cuestionario fue poco relevante siendo confiables los resultados, pues la consistencia interpretativa del coeficiente alfa de Cronbach fue de 0,7. Al aplicar la matriz DAFO para el análisis de vulnerabilidad se observó que los criterios de orden, de motricidad y dependencia estuvieron en el cuadrante conflicto lo cual se pudo interpretar como la presencia de condiciones de desconocimiento y estado técnico de las edificaciones en condiciones desfavorables. Se concluye que, la población no posee percepción del riesgo ante eventos naturales adversos como son los sismos. No han tenido capacitación u orientación ante las condiciones de vulnerabilidad de sus viviendas para actuar, ante una probable ocurrencia. Las condiciones de construcción técnica de las viviendas no cumplen con los requerimientos de resistencia ante los sismos.

Palabras clave: edificación; peligro; vulnerabilidad

\section{Abstract}

Earthquakes are adverse natural events that generate human losses and material damage. The aim of the study was to analyze seismicity and risk perception in the Department of Ica, Peru. The city of Pisco was selected because of the seismic event that occurred on August 15, 2007.

By means of random probability sampling, 10 houses at a distance of $1 \mathrm{~km}$ from each other and close to the coastline were selected for the vulnerability analysis. A survey was administered to one member of the

E-mail: pedro.doroteo@unica.edu.pe (c) Los autores. Este artículo es publicado por la Revista Campus de la Facultad de Ingeniería y Arquitectura de la Universidad
de San Martín de Porres. Este artículo se distribuye en los términos de la Licencia Creative Commons Atribución No-comercial
- Compartir-Igual 4.0 Internacional (https://creativecommons.org/licenses/ CC-BY), que permite el uso no comercial,
distribución y reproducción en cualquier medio siempre que la obra original sea debidamente citada. Para uso comercial
contactar a: revistacampus@usmp.pe.

https: 
household, corresponding to four of the 10 dwellings and the resident population's manifestation of seismic risk and housing vulnerability was analyzed. The Likert scale indicated that the knowledge of the population according to the questionnaire was not very relevant and the results were reliable, since the interpretative consistency of Cronbach's alpha coefficient was 0.7 . When applying the SWOT matrix for the vulnerability analysis, it was observed that the criteria of order, mobility and dependence were in the conflict quadrant, which could be interpreted as the presence of unfamiliar conditions and technical condition of the buildings in unfavorable conditions. It is concluded that the population does not have perception of risk in the face of adverse natural events such as earthquakes. They have not received training or orientation on the vulnerability of their homes to act in the event of a probable occurrence. The technical construction conditions of the houses do not meet earthquake resistance requirements.

Key words: building; danger; vulnerability

\section{Introducción}

Los eventos sísmicos generan el colapso de infraestructuras, además de pérdidas económicas y vidas humanas (Pnevmatikos et al., 2020). Entre las variables que se analizan según el riesgo sísmico, se encuentran la exposición al peligro y la vulnerabilidad de condiciones de escenario (Kang \& Kim, 2016; Shuai et al., 2020). Los terremotos se encuentran entre los eventos sísmicos más devastadores a nivel mundial. Desde 1998 hasta el 2018, los desastres por terremotos ocasionaron aproximadamente 752,498 personas muertas y 1.574 .000 lesionadas (EM-DAT, 2019). Aunque, los terremotos son eventos que ocurren con menos frecuencia, las consecuencias sociales, económicas y políticas son devastadoras en el tiempo (Barúa et al., 2020).

Cuando existen asentamientos cerca de fallas activas y se presentan debilidades estructurales en las viviendas, entonces la resistencia ante el movimiento sísmico resulta menor y, ello explica gran parte de la vulnerabilidad (Robinson, 2018). Al registrarse un sismo de gran magnitud, se observan daños significativos, se producen actividades de emergencia para la evacuación (Ej.: rutas de refugios, tipos refugios, horarios de salida, costo de evacuación, etc.), existe la movilidad de recursos asociados y necesarios con destino a un lugar seguro (Ej.: transporte) y que permanezcan en tal sitio para garantizar, el bienestar humano (Sadri et al., 2014; Lindell et al., 2015). Asimismo, debe resaltarse que el proceso de toma de decisiones tampoco es sencillo (Golshani et al., 2019), y por lo general, existe un enfoque de ciencias sociales para la garantía de evacuaciones (Wafda et al., 2013; Huang et al., 2017).

El Perú se encuentra en el Cinturón de Fuego del Pacífico, por tanto, existe probabilidad alta de sismos. Entre los sismos principales ocurridos en el territorio se encuentran los siguientes (Tabla 1): 
Tabla 1

Sismos en el Perú (últimos 50 años)

\begin{tabular}{ccl}
\hline Fecha & Epicentro & \multicolumn{1}{c}{ Magnitud } \\
\hline $31-05-1970$ & 7,8 & Callejón de Huaylas (Áncash) \\
$09-12-1970$ & 7,2 & Tumbes \\
$03-10-1974$ & 8,0 & Lima \\
$16-02-1979$ & 6,2 & Arequipa \\
$29-05-1990$ & 6,4 & San Martín, Amazonas, Cajamarca, Riojas, Moyobamba \\
$04-04-1991$ & 6,2 & Chachapoyas \\
\hline $12-11-1996$ & 6,4 & San Martín, Amazonas, La Libertad \\
$21-06-2001$ & 8,4 & Moquegua, Tacna, Arequipa \\
\hline $15-08-2007$ & 7,9 & Lima, Huancavelica, Junín, Ica, Ayacucho \\
$24-08-2011$ & 7,0 & Pucallpa \\
\hline $26-05-2019$ & 8,0 & Loreto \\
\hline
\end{tabular}

Tavera et al., (2007) indican que en el informe preliminar de la Dirección Sismológica del Instituto de Geofísica del Perú (IGP) se señala, que durante el sismo del 15 de agosto, los parámetros hipocentrales se calcularon mediante informaciones de la "Red Sísmica Nacional - IGP” perteneciente al IGP. Los valores fueron siguientes:

- Tiempo origen: $23 \mathrm{~h} 40 \mathrm{~m} 58.0 \mathrm{seg}$. (GMT, Hora Universal) $18 \mathrm{~h} 40 \mathrm{~m}$ $58.0 \mathrm{seg}$. (hora local)

- Latitud sur: $-13.67^{\circ}$

- Longitud oeste: $-76.76^{\circ}$

- Profundidad: $40 \mathrm{~km}$

- Magnitud: 7.0ML (Richter), 7.9Mw (magnitud momento)

- Intensidad máxima: VII (MM) en Pisco, Chincha, Cañete

- Momento sísmico: 1.2E+21 N-m (NEIC)

La Figura 1 muestra la distribución espacial de los sismos con magnitudes mayores a $4.0 \mathrm{ML}$ y que ocurrieron entre 1999 y el 2006 para aquellos de foco superficial (círculos $=$ menor a $60 \mathrm{~km}$ ) y de foco intermedio (cuadrados $=$ entre 61 y $350 \mathrm{~km}$ ).
En general, se observa que los sismos superficiales se distribuyen formando dos alineamientos importantes:

- 1ro), con epicentros entre la línea de fosa y de costa (asociado a la superficie de fricción de placas dentro del proceso de convergencia); y el

- 2do), en el interior del continente alineado en dirección NO-SE (origen en la reactivación esporádica de los principales sistemas de fallas geológicas presentes en el continente).

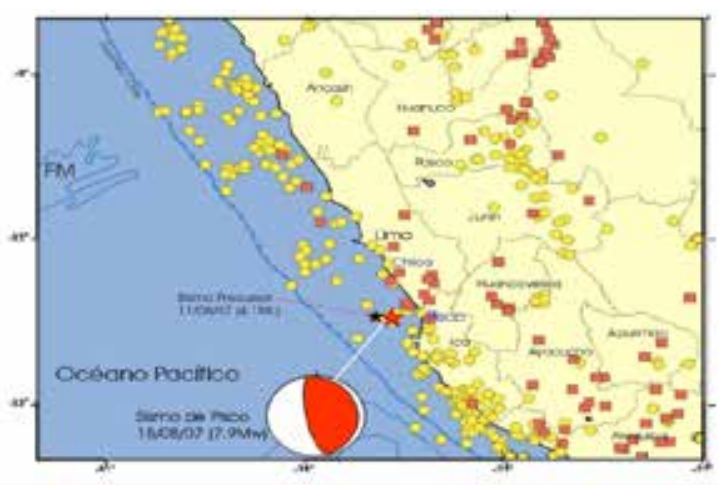

Figura 1. Distribución espacial de la sismicidad ocurrida en la región central de Perú entre los años 1999 y 2006 (ML>4.0) clasificada en sismos con foco superficial (círculos=menor a $60 \mathrm{~km}$ ) e intermedios (cuadrados, entre 61 y $350 \mathrm{~km}$ ).

Nota. Tavera et al., (2007). 
En general, se observa que los sismos superficiales se distribuyen formado dos alineamientos importantes:

- 1 ro. Con epicentros entre la línea de fosa y de costa (asociado a la superficie de fricción de placas dentro del proceso de convergencia) $\mathrm{y}$;

- 2do. En el interior del continente alineado en dirección NO-SE (origen en la reactivación esporádica de los principales sistemas de fallas geológicas presentes en el continente).

En el caso de los sismos de foco intermedio su patrón de distribución no es uniforme, pero existe mayor concentración en su ubicación para Chilca y Pisco, lo cual señala un incremento en la deformación para esta área a niveles de profundidad en el orden de 61 a $100 \mathrm{~km}$.

El sismo se caracterizó por indicar, un evento que precedió el día 11 de agosto (a las 19:18 horas) y con una magnitud de 4.1ML. Además, le sucedieron aproximadamente 40 réplicas. El epicentro del sismo se localizó a $77 \mathrm{~km}$ al noroeste de Pisco y no fue perceptible por la población ya que, al encontrarse la zona dentro del potencial sísmico, entonces no se considera el suceso como un indicador eminente de la probable ocurrencia por un sismo de mayor magnitud. El sismo de Pisco mostró dos características significativas:

- 1ro) magnitud y

- 2do) duración de registro (percepción del sismo por las personas)

En el caso de la duración, se asocia de manera directa al complejo proceso de ruptura que caracterizó al sismo y que puede ser, analizada en los registros de aceleración y desplazamiento del suelo que se obtienen a distancias regionales y telesísmicas. El registro de la aceleración del suelo correspondiente a la componente vertical de la estación Mayorazgo (MAY, $166 \mathrm{~km}$ de distancia epicentro-estación) donde se observa la presencia de dos grupos de ondas que llegan con una diferencia de tiempo en el orden alrededor de 70 segundos (Figura 2).

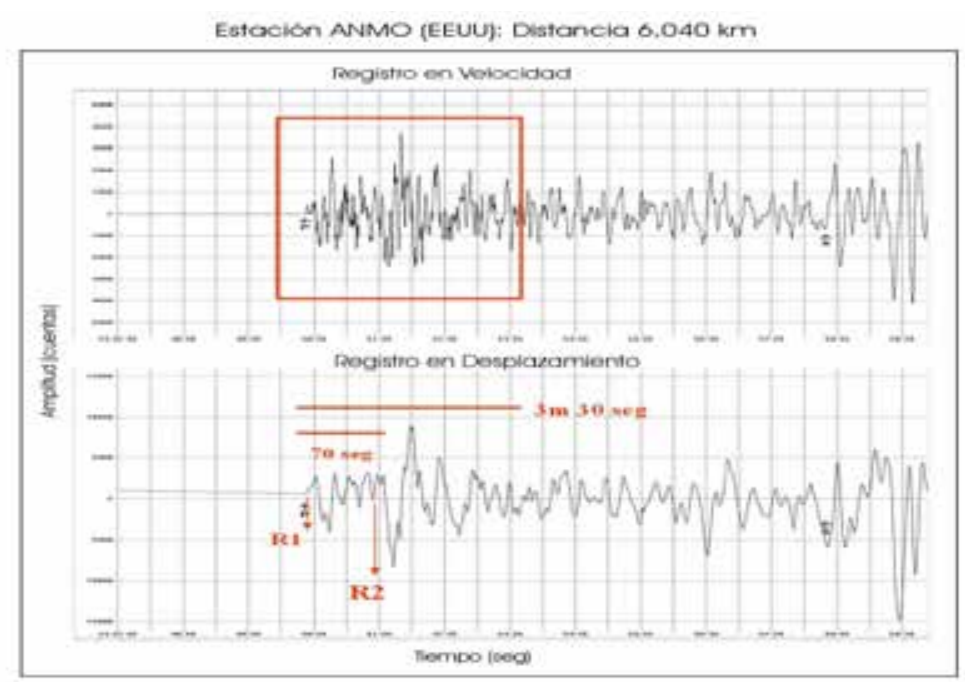

Figura 2. Registros de velocidad / desplazamiento del suelo / sismo de Pisco / estación ANMO (EEUU).

Nota. Tavera et al., (2007) 
El sismo de Pisco resultó el de mayor magnitud ocurrido en la región central de Perú hasta el 2007 durante los últimos 100 años (7.9Mw), y se caracterizó por presentar un proceso muy complejo de ruptura con propagación unilateral en sentido de dirección SE. Los dańos que se produjeron por el sismo fueron mayores en Pisco y algunas ciudades aledañas donde aquellas viviendas construidas de adobe y quincha, material noble con deficiencia estructural se afectaron.

Según, la escala Mercalli modificada, la intensidad máxima para la ciudad de Pisco fue de VII. De acuerdo a la ubicación hipocentro, el sismo de Pisco se originó con el mecanismo de fricción de placas dentro del proceso de convergencia que se desarrolló en el borde occidental de Perú. La orientación de los planos de falla y ejes de presión $(\mathrm{P})$ que se obtuvieron mediante el mecanismo focal fueron coherentes con la orientación de la fosa y con la dirección de convergencia de placas.

El sismo de Pisco presentó su epicentro en el área definida como laguna sísmica y que se encuentra ubicada entre las áreas de ruptura de los sismos de 1974 y 1996. El primero, afectó la ciudad de Lima y el segundo a la ciudad de Nazca. Finalmente, el asesoramiento técnico a la percepción pública, es uno de los criterios analíticos para evitar que se realice un inadecuado uso en la planificación del suelo y que luego, ante los movimientos sísmicos se produzcan los daños (Margaret \& Wendy, 2019; Bathrellos et al., 2019).

La percepción pública es un elemento de juicio ante la evaluación del riesgo (Dwyer et al., 2004), y en los últimos años, las investigaciones relacionadas con los terremotos se centran sobre crear conciencia y reducir los posibles efectos de futuros terremotos (De Pascale et al., 2017; Doyle et al., 2018; Han et al., 2020). El objetivo del estudio fue analizar la sismicidad y percepción del riesgo en el departamento de Ica, Perú.

\section{Métodos}

De las provincias del departamento de Ica se seleccionó a Pisco por su antecedente sismológico del 15 de agosto de 2007 donde se analiza la vulnerabilidad de las edificaciones. En el caso de la población residente, se seleccionó mediante un muestreo probabilístico aleatorio, 10 viviendas equidistantes a $1 \mathrm{Km}$. de distancia y cercanas a la línea de costa donde se les aplicó una encuesta solo a un miembro del núcleo familiar.

Se utilizó, el registro fotográfico para el análisis de las infraestructuras y sus condiciones de vulnerabilidad después de ocurrido el suceso sismológico donde se describe el estado físico, luego de 13 años transcurridos el evento adverso. Para el conocimiento sobre el mecanismo de protección y la manifestación de la población residente se aplicó una encuesta.

Se elaboró una escala Likert (Tabla 2) con cinco criterios donde se asignó códigos de puntuación desde 1 hasta 4 y luego se aplicó para la confiablidad de los datos, el coeficiente alfa de Cronbach (1951): utilizando el método de la varianza de los criterios según la siguiente expresión:

$$
\alpha=\mathrm{K} / \mathrm{K}-1\left[1-\sum \mathrm{V} 1 / \mathrm{Vt}\right]
$$

\footnotetext{
Donde:

- $\alpha=$ alfa de Cronbach; $\mathrm{K}$ = número de items; V1 = varianza de cada items; $\mathrm{Vt}=$ varianza total
} 
Tabla 2

Escala tipo Likert

\begin{tabular}{|c|c|c|c|}
\hline \multicolumn{4}{|c|}{ Vulnerabilidad socioambiental } \\
\hline$\underset{4}{\text { Muy alto }}$ & $\begin{array}{c}\text { Alto } \\
3\end{array}$ & $\begin{array}{c}\text { Bajo } \\
2\end{array}$ & $\begin{array}{c}\text { Muy bajo } \\
1\end{array}$ \\
\hline \multicolumn{4}{|c|}{ Responsabilidad social-ambiental } \\
\hline Muy alto & $\begin{array}{c}\text { Alto } \\
3\end{array}$ & Bajo & Muy bajo \\
\hline \multicolumn{4}{|c|}{ Presencia estacionaria de monitoreo y registro } \\
\hline $\begin{array}{c}\text { Muy alto } \\
4\end{array}$ & $\begin{array}{c}\text { Alto } \\
3\end{array}$ & $\begin{array}{c}\text { Bajo } \\
2\end{array}$ & $\begin{array}{c}\text { Muy bajo } \\
1\end{array}$ \\
\hline \multicolumn{4}{|c|}{ Validez de tecnografías locales } \\
\hline $\begin{array}{c}\text { Muy alto } \\
4\end{array}$ & $\begin{array}{c}\text { Alto } \\
3\end{array}$ & $\begin{array}{c}\text { Bajo } \\
2\end{array}$ & Muy bajo \\
\hline \multicolumn{4}{|c|}{ Programas de prevención ante eventos naturales adversos } \\
\hline $\begin{array}{c}\text { Muy alto } \\
4\end{array}$ & $\begin{array}{c}\text { Alto } \\
3\end{array}$ & $\begin{array}{c}\text { Bajo } \\
2\end{array}$ & $\begin{array}{c}\text { Muy bajo } \\
1\end{array}$ \\
\hline
\end{tabular}

Se analizó mediante la matriz DAFO y influencia de los ítems sobre la predicción luego, el método de los cuadrantes, la de conflictos socio-ambientales (Tabla 3).

Tabla 3

Matriz DAFO = debilidad, amenaza, fortaleza, oportunidad

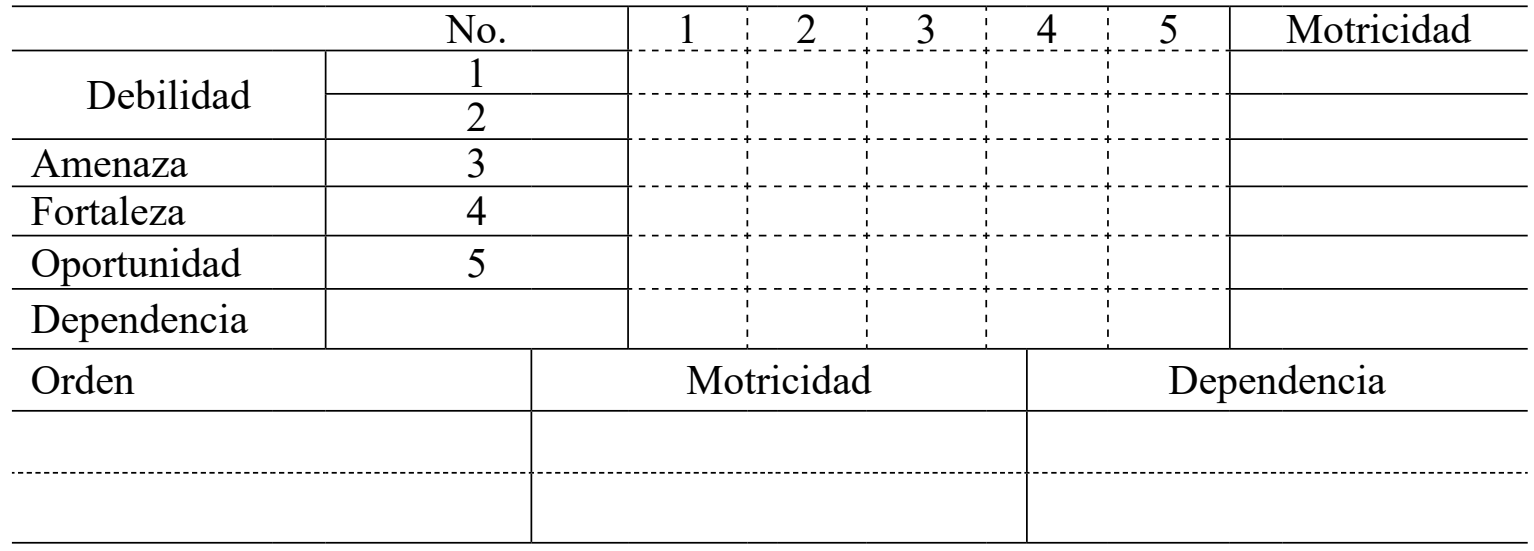

Métodos de los cuadrantes

\begin{tabular}{l|l|l|l|l|l}
\hline & & & & \\
\hline & poder & & & & \\
\hline & & & & & \\
\hline & autonomía & & & & \\
\hline & & & & & \\
\hline
\end{tabular}

Se utilizó el programa estadístico profesional Epidat 4.2 (versión libre) donde se describió el promedio, análisis de la varianza y el contraste de hipótesis mediante el análisis t para una muestra. Los resultados se consideran significativos cuando $\mathrm{p}<0,05$.

\section{Resultados y Discusión}

La Tabla 4 muestra, la escala tipo Likert (calificación numérica) para los cinco criterios que se indicaron ante el marcaje de las personas que se encuestaron. 
Tabla 4

Evaluación / Escala tipo Likert

\begin{tabular}{ccccccc}
\hline $\begin{array}{c}\text { Escala de } \\
\text { valor }\end{array}$ & $\begin{array}{c}\text { Vulnerabilidad } \\
\text { socioambiental }\end{array}$ & $\begin{array}{c}\text { Responsabilidad } \\
\text { social-ambiental }\end{array}$ & $\begin{array}{c}\text { Presencia } \\
\text { estacionaria } \\
\text { de monitoreo } \\
\text { y registro }\end{array}$ & $\begin{array}{c}\text { Validez de } \\
\text { tecnografías } \\
\text { locales }\end{array}$ & $\begin{array}{c}\text { Programas de } \\
\text { prevención } \\
\text { ante eventos } \\
\text { naturales } \\
\text { adversos }\end{array}$ \\
\hline 1 & 3 & 4 & 4 & 4 & 4 & 19 \\
2 & 3 & 4 & 3 & 3 & 4 & 17 \\
\hline 3 & 3 & 3 & 3 & 3 & 3 & 15 \\
4 & 4 & 4 & 3 & 4 & 3 & 18 \\
\hline Promedio & 3,25 & 3,75 & 3,25 & 3,5 & 3,5 & 17,25 \\
\hline
\end{tabular}

La Tabla 5 muestra el criterio que se otorgó ante los indicadores donde se evaluó las respuestas de la encuesta y que resultó un conocimiento poco relevante.

Tabla 5

Criterio según los indicadores

\begin{tabular}{c|c|c|c|c}
\hline \multirow{2}{*}{ Indicadores } & \multicolumn{4}{|c}{ Criterio } \\
\cline { 2 - 5 } & Muy alto & Alto & Bajo & Muy bajo \\
\cline { 2 - 5 } & 4 & 3 & 2 & 1 \\
\hline 1 & 3,8 & 3,4 & & \\
2 & & 3,0 & & \\
3 & & & & \\
4 & 3,6 & & & \\
\hline
\end{tabular}

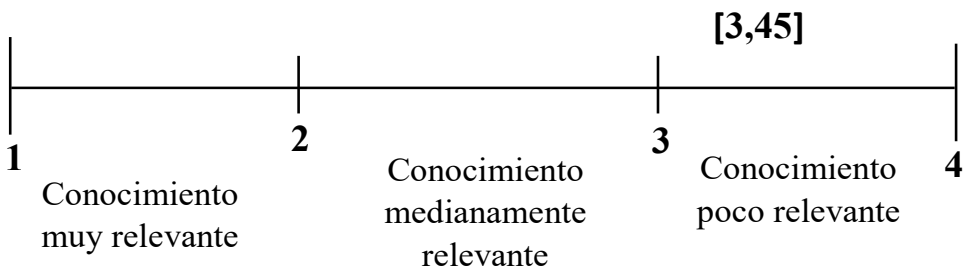

Al realizar el análisis de varianza no se halló diferencias estadísticas significativas $(\mathrm{p}=0,2463)$ con relación a las respuestas de los encuestados. La Figura 3 muestra la distribución $t_{\text {tab }}$ promedio de respuestas con relación a las preguntas efectuadas.

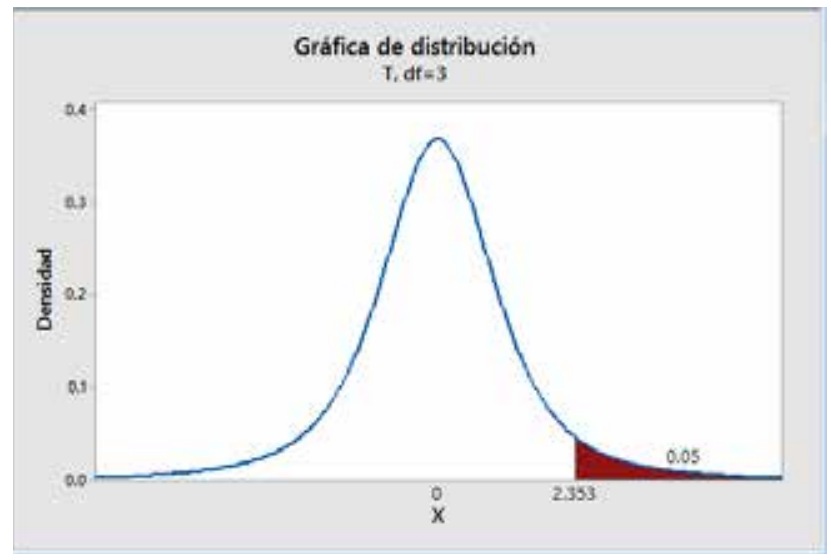

Figura 3. Distribución $t_{\text {tab }} /$ promedio de calificaciones / encuestados 
La estadística básica para $\mathrm{t}$ de 1 muestra, describió lo siguiente:

- Hipótesis nula $-\mathrm{H}_{0}: \mu=3$

- Hipótesis alterna $-\mathrm{H}_{1}: \mu>3$; valor Tcal $=2,33$; valor $\mathrm{p}=0,051$
De modo que, al ser $2,33<2,353$ se acepta la hipótesis nula $(\mathrm{Ho})$ y donde se plantea la búsqueda de propuestas para generar confiabilidad (Tabla 6).

Tabla 6

Estadistica descriptiva / respuesta

\begin{tabular}{ccccc}
\hline Entrevistados & Media & $\begin{array}{c}\text { Desviación } \\
\text { Estándar }\end{array}$ & $\begin{array}{c}\text { Error estándar } \\
\text { de la media }\end{array}$ & $\begin{array}{c}\text { Límite inferior de 95\% para } \\
\mu\end{array}$ \\
4 & 3,438 & 0,375 & 0,187 & 2,996 \\
\hline
\end{tabular}

La Tabla 7 indica la confiabilidad de los datos que se midieron mediante el alfa de Cronbach para los notarios.

Tabla 7

Confiabilidad de los datos $/ V=$ varianza $/$ alfa de Cronbach

\begin{tabular}{|c|c|c|c|c|c|c|c|}
\hline $\begin{array}{l}\text { Entrevis- } \\
\text { tados }\end{array}$ & $\begin{array}{l}\text { Vulnera- } \\
\text { bilidad } \\
\text { socio- } \\
\text { ambiental }\end{array}$ & $\begin{array}{l}\text { Responsa- } \\
\text { bilidad } \\
\text { social- } \\
\text { ambiental }\end{array}$ & $\begin{array}{c}\text { Presencia } \\
\text { estacionaria } \\
\text { de monitoreo } \\
\text { y registro }\end{array}$ & $\begin{array}{l}\text { Validez } \\
\text { de tecno- } \\
\text { grafías } \\
\text { locales }\end{array}$ & $\begin{array}{l}\text { Programas de } \\
\text { prevención } \\
\text { ante eventos } \\
\text { naturales } \\
\text { adversos } \\
\end{array}$ & $\sum \mathrm{V}$ & $\mathrm{Vt}$ \\
\hline 4 & 0,5 & 0,5 & 0,5 & 0,58 & 0,58 & 2,66 & 1,25 \\
\hline Indicador & Valor & & & & & & \\
\hline K & 5 & & & & & & \\
\hline$\sum \mathrm{V}$ & 2,66 & & & & & & \\
\hline $\mathrm{Vt}$ & 1,70 & & & & & & \\
\hline \multicolumn{8}{|c|}{$\begin{array}{l}\alpha=5 / 5-1[1-2,66 / 1,70] \\
\alpha=1,25[1-1,56] \\
\alpha=1,25[0,56] \\
\alpha=0,7\end{array}$} \\
\hline
\end{tabular}

La Tabla 8 muestra la asignación de puntuación según el alfa de Cronbach con relación a la evaluación de las respuestas de los encuestados. Dado que su valor fue 0,7 se validó la encuesta para su consideración lo cual se corrobora, la preocupación de la población residente ante la ocurrencia de nuevos eventos naturales adversos como los sismos. 
Tabla 8

Consistencia interpretativa / coeficiente alfa de Cronbach

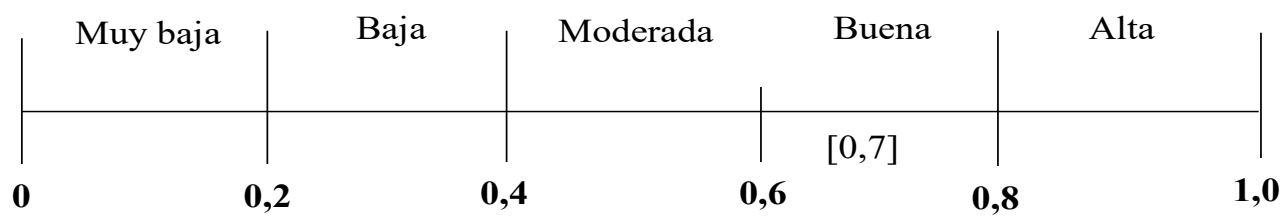

La Figura 4 muestra algunas de las viviendasqueal parecer fueron construidas sin ningún tipo de supervisión profesional y donde se combinan materiales de paredes de caña y barro, mampostería de ladrillo de adobe, escalera de concreto (colapsada), aligerado de concreto que son vulnerables ante la ocurrencia de eventos sísmicos.

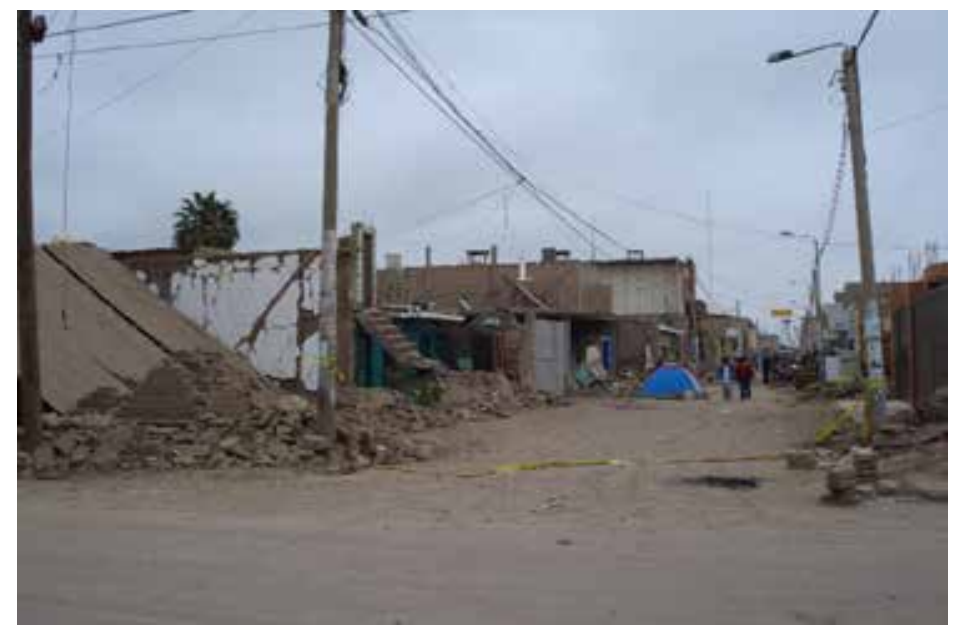

Figura 4. Construcción de viviendas sin supervisión profesional

La Figura 5 muestra el colapso de las viviendas por el uso inapropiado y combinatorio de materiales inseguros (adobe, muro de ladrillos pandereta en primer y segundo nivel). Se aprecia la cimentación superficial y la separación entre columnas no acorde a lo permisible.

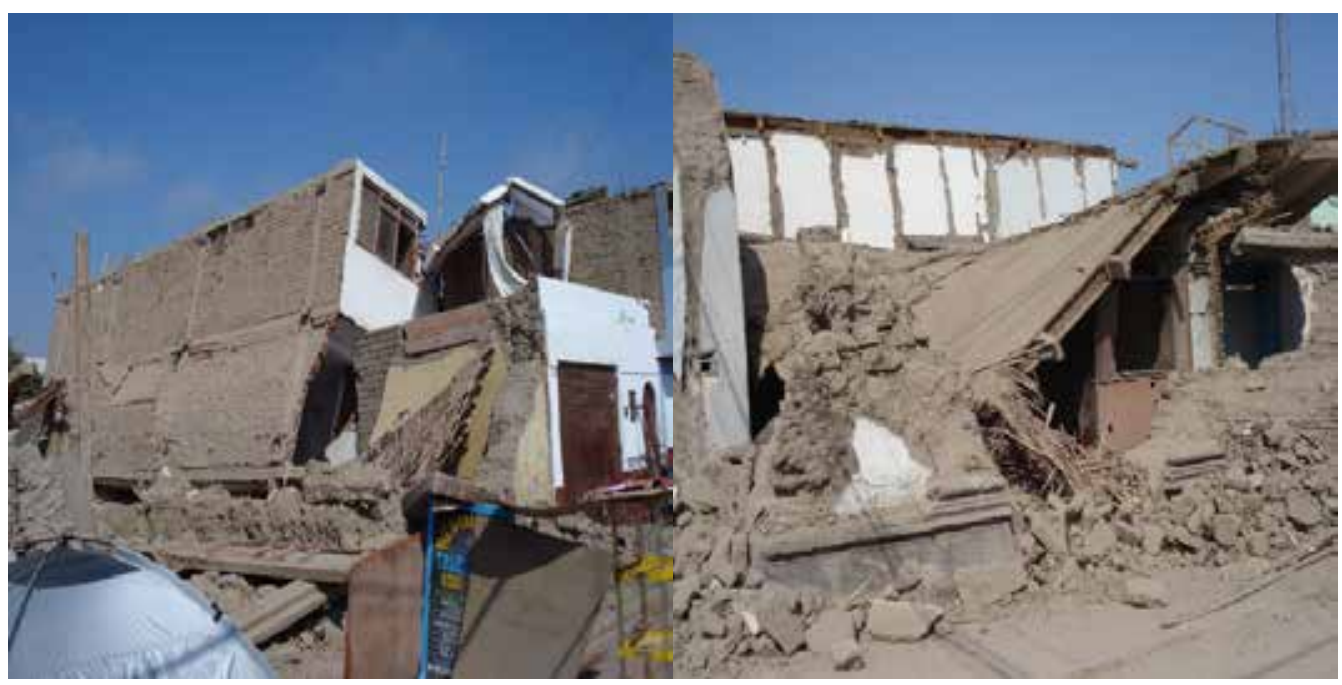

Figura 5. Edificación con deficiencia estructuración de columnas 
La Figura 6 muestra edificaciones denominadas autoconstrucciones $\mathrm{y}$ donde existen deficiencias técnicas, el completo para determinada altura de piso a techo con ladrillo e incluso enchapa la fachada sin amarre adecuado en las esquinas. El piso blando en aquellos edificios donde se observa en la planta baja que solo existen elementos verticales o columnas a este nivel, y en los pisos superiores se construyen cerramientos para viviendas con mampostería, lo cual hace estructuralmente que la planta baja tenga menor rigidez en comparación a los pisos superiores (lo sísmico resistente correcto, sería lo contrario, mayor rigidez en las plantas inferiores).

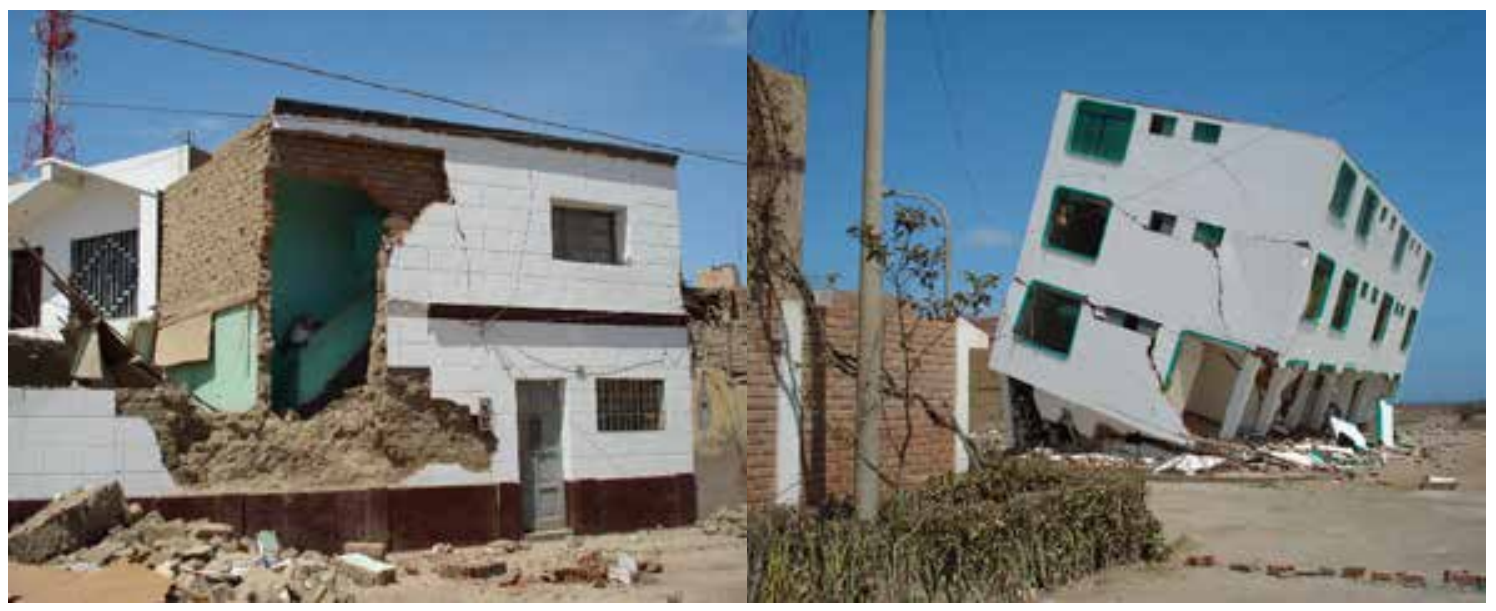

Figura 6. Autoconstrucciones de edificaciones

La Figura 7 muestra el Hospital de Pisco que es una edificación antigua con ladrillo hueco de cemento, alturas entre piso y techo alto lo que genera esbeltez, con luces intermedias (ventanas), confinamiento adecuado, luces libres sin apoyos intermedios, pero se aprecia entre las luces, el confinamiento solo con ladrillos lo que provocó a la edificación ser susceptible de colapsar ante el evento sísmico.

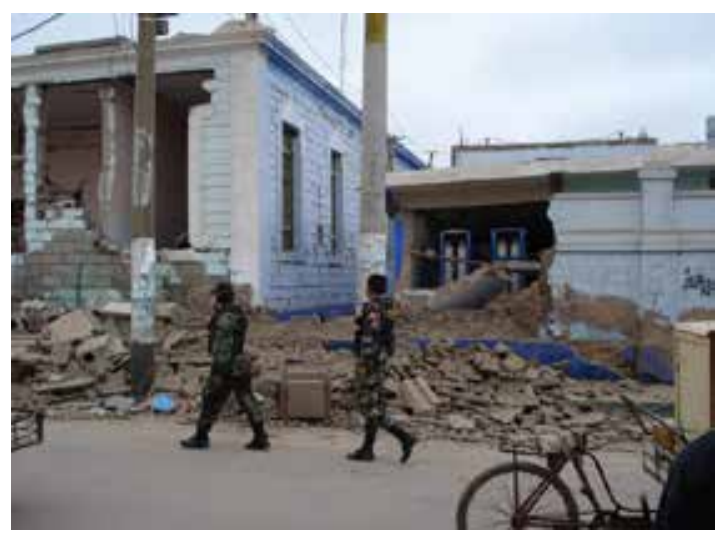

Figura 7. Hospital de la Ciudad de Pisco / confinamiento solo con ladrillos
Se observa el daño a la cúpula del Municipio de Pisco, declarado Monumento Histórico y en el cual colapsó la parte superior que fue construida con una losa delgada de concreto y malla de fierro donde se mantuvo el resto de la estructura (Figura 8).

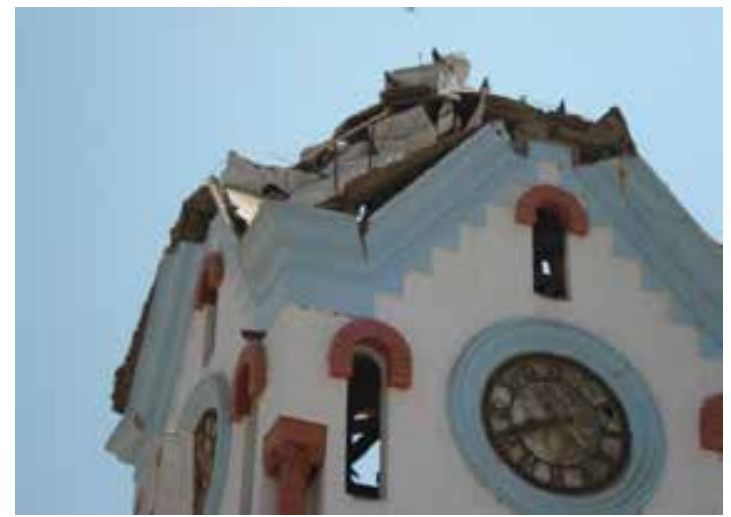

Figura 8. Fallas en los muros por corte

Para la elaboración de la matriz DAFO se asignó el siguiente criterio de puntuación a los indicadores: 
- Vulnerabilidad socioambiental (1)

- Responsabilidad social-ambiental (2)

- Presencia estacionaria de monitoreo y registro (3)

- Validez de tecnografías locales (4)
- Programas de prevención ante eventos naturales adversos (5)

La Tabla 9 indica la elaboración de la matriz DAFO y la relación que existió en los cuadrantes según indicadores descritos.

Tabla 9

Matriz DAFO / operacionalizada

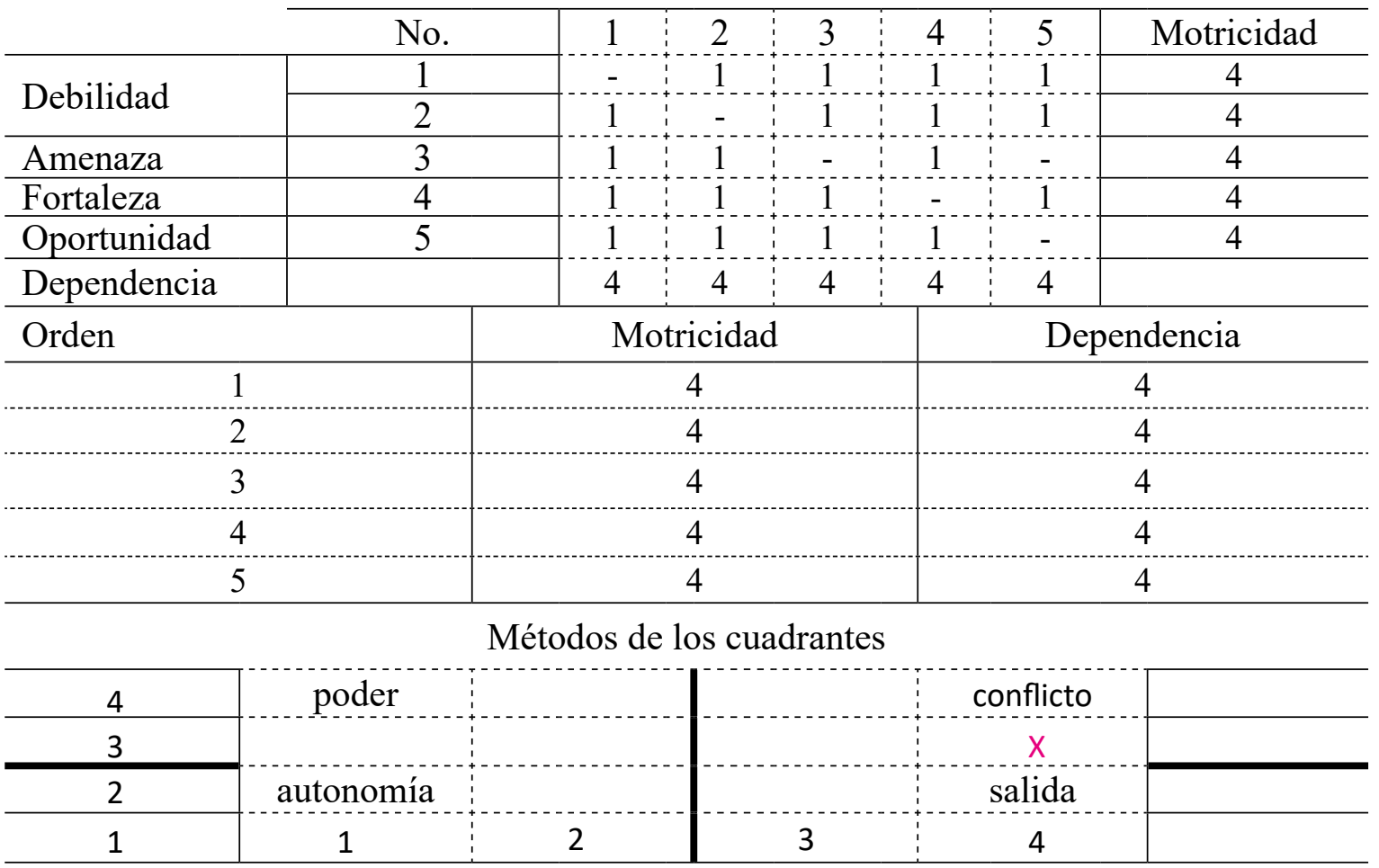

Una gran parte de la población mundial vive en zonas o áreas urbanas donde alrededor del $90 \%$ se encuentran en países con ingresos bajos, pero lo más preocupante radica, en el ritmo acelerado de expansión que este estrato evidencia (alrededor de 1,3 millones) por semana (UNDESA, 2015).

La concentración de vidas humanas y ciertos activos en estas áreas urbanas hace que la exposición a riesgos naturales adversos a las personas sea con más frecuencia y en consecuencia de manera comparada, el peligro aumenta mucho más que aquellas áreas rurales (Bilham,
2009). Es por ello, que la gran prioridad en los últimos tiempos obedezca a salvar la vida humana como a la protección de infraestructuras, pero estas cifras pueden ser inciertas, debido a que los datos del Banco Mundial señalan un porcentaje alrededor del $67 \%$ que aún, permanece en zonas rurales e incluso, la ONU señaló en el 2008 que la mitad de la población en Asia y África continúa viviendo en estas áreas rurales cuyo sustento es la agricultura (Li, 2014).

Por lo general, siempre se argumenta el enfoque de la vulnerabilidad y sus consecuencias en las áreas urbanas 
donde la extensión que presenta puede ser tan devastadora que sería imposible subestimar. Sin embargo, Wyss (2018) señala que los terremotos de mayor magnitud ocurren en zonas rurales, y por lo general más del $85 \%$ de las muertes obedecen a personas rurales. Lo que las personas piensan sobre el riesgo de los terremotos es de gran relevancia no solo para las personas en sí, sino para los que toman decisiones sobre los aspectos de gestión de riesgo y en la implementación sobre el código de construcción. Asimismo, un elemento fundamental es el comportamiento de las personas en el hogar y la comunidad cuando se produce el evento adverso natural como un sismo (Rohrmann, 1994).

Ante la percepción del riesgo por la ocurrencia de un evento adverso natural como un sismo, las personas señalan que existe elevada vulnerabilidad dada las condiciones de vida. No existe responsabilidad social-ambiental por parte de los tomadores de decisiones que apoyen sus solicitudes, tampoco una estación de registro que permita obtener resultados confiables, pues no se cuenta con las tecnologías y aquellos probables registros no son de la localidad por cuanto, si se produjera una catástrofe, la población con bajo nivel de ingreso no cuenta con un programa de prevención que les resguarde la vida y sus bienes materiales.

Recientemente, se han comenzado a investigar las actitudes populares en los países deingresosbajosyen lo fundamental para aquellos que experimentan cierto grado de desarrollo económico. Hace más de 15 años, Bronfman \& Cifuentes (2003) señalan que un estudio realizado en Chile como país emergente, pudo entenderse la mayor preocupación de su población por las amenazas y su demanda hacia el control y la regulación.

En otros estudios, se indican que las ciudades son el principal eje o motor de desarrollo económico donde la industria, infraestructura y la propia población se concentran de manera desproporcionada. Por ejemplo, Estambul alberga aproximadamente una octava parte de la población total de Turquía y representa la mitad de todo su potencial industrial (Erdik \& Durukal, 2008). Esta ciudad al igual que Pisco, se encuentra ubicada en las proximidades de fallas activas, pero a diferencia de Estambul, Pisco no es capital y menos existe un desarrollo industrial de significación. Por lo cual, y en proporción ante cualquier tragedia o desastre natural (England \& Jackson, 2011), no sería tan preocupante, pero no debe menospreciarse lo que sucedió, ya que su impacto a nivel local produjo más que la propia pérdida de materiales, un estado psico-estresante en la población. Aunque, hubo un elevado riesgo, todavía poco se conoce sobre la comprensión individual y familiar del riesgo que generó el terremoto o los niveles de preparación preventiva en los hogares y la comunidad.

Prácticamente, no existen estudios sociales que aborden tal problemática y existe la alta probabilidad que, de ocurrir nuevamente, se producirían dańos similares. Si bien se conoce, que los estudios de percepción de terremotos están disponibles o se limitan al propio entorno urbano, poca evidencia de los niveles crecientes de conciencia o preparación frente al riesgo continúan presentes (Mussakulova, 2017). Asimismo, la organización social 
al parecer, es quien determina que riesgo se puede priorizar una vez transcurrido el terremoto pero, indiscutiblemente la percepción del riesgo parece estar asociada a los valores culturales y educacionales ante la probable ocurrencia de un evento adverso como es un sismo.

Las percepciones de riesgo son particularmente difíciles de medir cuando se trata de peligros como sismos y que son de baja frecuencia, pero de gran magnitud. Las personas tienden a descartar los riesgos que se perciben como incontrolables, tienen potencial catastrófico o resultan en consecuencias fatales y que "se niegan a preocuparse por pérdidas cuya probabilidad está por debajo de algún umbral" e "ignoran las amenazas raras". Una mejor comprensión de la percepción del riesgo sísmico es la argumentación, lo que resulta esencial para desarrollar planes apropiados de comunicación del riesgo sísmico y adoptar medidas proactivas para la reducción del riesgo (Vicente et al., 2014).

Como muestra Covello (2010), existen muchos riesgos que preocupan y molestan a las personas, pero causan poco daño y también existen muchos riesgos que matan o mutilan a muchas personas, pero que no les preocupan ni trastornan indebidamente. A pesar de la necesidad de una mejor gestión de desastres para minimizar el riesgo de terremotos, todavía no se percibe el riesgo, incluso las políticas y procedimientos mejor diseñados pueden no conducir a los resultados deseados y entre las principales razones están las siguientes:

1. La percepción pública del riesgo es una parte esencial del riesgo de desastres (Santos et al., 2017), y
2. Los responsables políticos no incluyen las opiniones de la población sobre la percepción del riesgo en la formulación de las políticas públicas.

Saber, cómo el público percibe que el riesgo es importante, ya que proporciona una información sobre cómo y por qué las personas responden a los peligros de la forma en qué hacer (Bodoque et al., 2019). Para garantizar un impacto limitado de los terremotos, existe la necesidad de comprender qué factores afectan la subjetividad de un individuo y qué acciones les ayudarán a afrontar mejor un evento como los terremotos. Entender tales influencias permitirá a las políticas y la propia práctica centrarse en garantizar que se pongan en acción las estrategias adecuadas en el propio lugar. Sin embargo, la percepción del riesgo es altamente interpretativa debido a su proceso dinámico sobre el cual los expertos en gestión de desastres y el público en general, a menudo tienen una comprensión diferente de las amenazas y los riesgos (Sjoberg, 1999).

Si bien la percepción pública del riesgo, generalmente, está impulsada por el interés económico, los sesgos intuitivos y los valores culturales, el riesgo de los expertos ante la percepción tiende a ser más un producto analítico, objetivo y racional que las mismas evaluaciones de riesgos (Kasperson et al., 2000).

Después del terremoto de Pisco, uno de los problemas más notables fue el relacionado con los efectos geotécnicos ligados a la estructura geológica de la región, evidenciándose los desplazamientos del suelo, así como la licuefacción del suelo (fenómeno en el 
cual los terrenos, a causa de saturación de agua y particularmente en sedimentos como arena o grava, pierden su firmeza y fluyen como resultado de los esfuerzos provocados por los sismos). Asimismo, tal fenómeno al producirse hace que las edificaciones sufran asentamientos diferenciales que podrían ser de $0.1 \mathrm{~cm}$, y llegar hasta $1.00 \mathrm{mt}$. Esta situación determina que a causa de un evento sísmico, las viviendas pierdan estabilidad y terminen colapsando.

Se observó que las construcciones eran antiguas y muchas de ellas de material de adobe que representa muy baja resistencia ante la presencia de un evento sísmico. Por esa razón, puede decirse que este tipo de material puede soportar esfuerzos a la compresión (esfuerzo al que está sometido un cuerpo por la aplicación de fuerzas que actúan en el mismo sentido), pero se vuelve muy vulnerable ante las fuerzas de tracción (cuando las fuerzas con la misma dirección y de sentidos contrarios tienden a estirarlo). Es por ello, que ante el evento sísmico se superó la tracción que pudo suportar el muro de adobe donde finalmente se destruyó. Las construcciones con muros de adobe tenían una baja adherencia y fueron construidas sin ningún tipo de refuerzo permitido por cuanto, no hubo integridad; sin embargo, al no existir refuerzos se produjo el colapso, y en consecuencia la caída de los techos.

No solo de material de adobe consistieron las edificaciones. También, se observó que colindantes con ellas se construyeron casas de quincha en el segundo nivel de construcción, lo que justificaría su integración en comparación con el primer nivel de adobe. La quincha está constituida por estructuras de caña que son elementos delgados, flexibles y amarrados entre sí, con cuero de animales vacunos que se remojan y tejen. $\mathrm{Al}$ secarse este cuero logra fijar las cañas dentro de marcos de madera lo que hace a diferencia de la edificación de adobe, se absorban los efectos de tracción, originándose en estas edificaciones que "sufra" ante la presencia de grietas superficiales, $y$ desprendimiento del barro que sirve como tarrajeo, pero sí, se mantienen ante un evento sísmico.

Se pudo observar que el suelo de Pisco es un suelo blando, lo que hace que las edificaciones colapsen repentinamente ante un evento sísmico, y ello fue uno de los factores que hizo que se perdieran muchas vidas porque no les dio el tiempo necesario para evacuarse a otros sitios seguros. Muchas de las viviendas de albañilería presentaron una serie de deficiencias, ya que en su ejecución no se tuvieron en cuenta las disposiciones y limitaciones que establecen las normas de diseño y cálculos correspondientes especialmente en las aberturas (puertas y ventanas) muchas de ellas sin ningún tipo de reforzamiento.

Otro daño observado y que se notó concurrente fue la falta de confinamiento (viga collarín), en los bordes superiores de los muros para pisos superiores. Por lo general, el segundo piso de las viviendas era de tipo unifamiliar donde la falta de muros hace que se produzca en el pańo, una grieta en forma perpendicular a su plano. En los diseños arquitectónicos desarrollados para las edificaciones de material noble, no se tuvo en cuenta aspectos de orden técnico constructivos, no se consideró la capacidad portante del suelo. Se construyeron edificaciones en las que en el primer nivel solo estaban sostenidas por columnas sin ningún 
tipo de cerramiento de muro, y lo que al parecer fue más dañino, existió encima de estas edificaciones un cerramiento de columnas y muros que produjeron una rigidez baja y/o resistencia.

Otro de los defectos de carácter constructivo que se apreció después del sismo fue la configuración vertical de sus edificaciones ya que estas presentaban irregularidades por los cambios bruscos de rigidez y masa entre pisos consecutivos, lo que ocasionó en el evento sísmico, fuertes concentraciones de esfuerzos como se pudo observar en el edificio que se proyectó hacia adelante. Esta edificación, aparentemente de albañilería reforzada, muestra su reducida densidad de muro, especialmente, en la dirección paralela a la fachada y esta limitación se vuelve más crítica en la medida que el número de pisos aumenta. Es decir, puede observarse una serie de causas que condicionaron la pérdida de las viviendas como son los deficientes diseños, incorrectos elementos constructivos y violación de procedimientos para la supervisión.

Se observó que la cúpula de la Municipalidad de Pisco fue fabricada con losas de concreto a manera de losa con refuerzo de fierro, la misma que se encontraba dañada por la corrosión. Además, las losas se observaron delgadas, quizás con el propósito de disminuir el peso a la estructura; sin embargo, estas no soportaron los efectos del sismo y colapsaron.

Robinson (2018) señala que, en la propia urbanización, igualmente existe vulnerabilidad en la población cuando los asentamientos se encuentran cercanos a las fallas activas y existe debilidad estructural en las viviendas.

Se concluye, que la población no posee percepción del riesgo ante eventos naturales adversos como son los sismos y donde se menciona la ausencia de capacitación u orientación ante las condiciones de vulnerabilidad de sus viviendas para actuar ante una probable ocurrencia. De igual modo, las condiciones de construcción técnica de las viviendas no cumplen con los requerimientos de resistencia ante la probable ocurrencia de un evento natural adverso como los sismos. Aunque los daños causados en derrumbes fueron cuantificados debe señalarse la gran preocupación por el incremento en las mismas condiciones de construcción de viviendas y que las hacen del mismo modo vulnerable.

\section{Referencias}

Bathrellos, D. George, D. \& Skilodimou, H. (2019). Land use planning for natural. Hazards Land; 8(9), 128. http://dx.doi.org/10.3390/ land 8090128

Bilham, R. (2009). The seismic future of cities. Bull. Earthq. Eng; 7, 839887. http://dx.doi.org/10.1007/ s10518-009-9147-0
Bodoque, J.M., Díez, H.A., Amerigo, M., García, J.A. \& Olcina, J. (2019). Enhancing flash flood risk perception and awareness of mitigation actions through risk communication: a pre-post survey design. J. Hydrol; 568, 769-779. http://dx.doi.org/10.1016/j. jhydrol.2018.11.007 
Bronfman, N. \& Cifuentes, L. (2003). Risk perception in a developing country: the case of Chile Risk Anal; 23(6), 1271-1285. https://doi.org/10.1111/j.02724332.2003.00400.x

Covello, V.T. (2010). Strategies for overcoming challenges to effective risk communication. In: Heath, R.L., O'Hare, H.D. (Eds.), Handbook of Risk and Crisis Communication. Routledge, New York, pp. 143-167.

Cronbach, L.J. (1951). Coefficient alpha and the internal structure of tests. Psychometrika, 16: 297 334. https://doi.org/10.1007/ BF02310555

De Pascale, F., Bernardo, M., Muto, F., Di Matteo, D. \& Dattilo, V. (2017). Resilience and seismic risk perception at school: a geoethical experiment in Aiello Calabro, southern Italy. Nat. Hazards; 86(2), 569-586. https://doi.org.10.1007/ s11069-016-2696-z

Doyle, E.E., McClure, J., Potter, S.H., Becker, J.S., Johnston, D.M., Lindell, M.K. \& Coomer, M.A. (2018). Motivations to prepare after the 2013 cook strait earthquake, NZ. Int. J. Disaster Risk Reduct, 31, 637-649. http://dx.doi. org/10.1016/j.ijdrr.2018.07.008

Dwyer, A., Zoppou, C., Nielsen, O., Day, S. \& Roberts, S. (2004). Quantifying social vulnerability: a methodology for identifying those at risk to natural hazards. Geoscience Australia; 22-23. ISBN: 1-920871-09-8
EM-DAT (2019). The Emergency Events Database - Universite Catholique de Louvain (UCL) - CRED, D. Guha-Sapir, 2019. Brussels, Belgium). https://public.emdat.be/

England, P. \& Jackson. J. (2011). Uncharted seismic risk. Commentary Nat. Geosci; 4, 348-349. https:// doi.org/10.1038/ngeo1168

Erdik, M. \& Durukal, E. (2008). Earthquake risk and its mitigation in Istanbul. Nat. Hazards; 44, 181197. http://dx.doi.org/10.1007/ s11069-007-9110-9

Golshani, N.; Shabanpour, R.; Mohammadian, A.; Auld, J. \& Ley, H. (2019). Evacuation decision behavior for no-notice emergency events. Transport. Res. Part D-Transp. Environ; 77, 364377. https://doi.org/10.1016/j. $\operatorname{trd} .2019 .01 .025$

Han, Z., Wang, L. \& Cui, K. (2020). Trust in stakeholders and social support: risk perception and preparedness by the Wenchuan earthquake survivors. Environ. Hazards; 1-14. https://doi.org/10. 1080/17477891.2020.1725410

Wafda, F., Saputra, R.W., Nurdin, Y., Nasaruddin, S. \& Munadi, K. (2013). Agent-based tsunami evacuation simulation for disaster education.InternationalConference on ICT for Smart Society. 1-4. http://dx.doi.org/10.1109/ ICTSS.2013.6588087

Huang, S.K.: Lindell, M.K. \& Prater, C.S. (2017). Multistage model 
of hurricane evacuation decision: empirical study of Hurricanes Katrina and Rita. Nat. Hazard. Rev; 18(3), 15.

Kang, H.S. \& Kim, Y.T. (2016). The physical vulnerability of different types of building structure to debris flow events. Natural Hazards; 80, 1475-1493. https://doi. org/10.1007/s11069-015-2032-z

Kasperson, R.E., Renn, O., Slovic, P. \& et al., (2000). The social implication of risk: a conceptual framework, in: P. kas (Ed.), The Perception of Risk, Earthscan Publications, London, 232-245.

Li, T.M. (2014). Land's End: Capitalist Relations on an Indigenous Frontier. Duke University Press, London.

Lindell, M.K.; Prater, C.S.; Gregg, C.E.; Apatu, E.J.I.; Huang, S.K. \& Wu, H.C. (2015). Households' immediate responses to the 2009 American Samoa Earthquake and Tsunami. Int. J. Disaster Risk Reduct, 12, 328-340. https://doi. org/10.1016/j.ijdrr.2015.03.003

Margaret, K. \& Wendy, S. (2019). Gaining public input on natural hazard risk and land-use planning, Disaster Prev. Manag. Int. J; 28, 228-244. https://doi.org/10.1108/ DPM-04-2018-0134

Mussakulova, M. (2017). L'analyse de la gestion du risque de laves torrentielles dans le contexte d'une ville soumise a ' de fortes contraintes environnementales et a 'des facteurs de vuln'erabilit'e croissants: l'exemple de la ville d'Almaty (Kazakhstan). PhD. Universit'e Lille - Sciences et Technologies. https://pepite-depot.univ-lille.fr/ LIBRE/EDSESAM/2017/503762017-Mussakulova.pdf

Pnevmatikos, N., Konstandakopoulou, F. \& Koumoutsos, N. (2020). Seismic vulnerability assessment and loss estimation in Cephalonia and Ithaca islands, Greece, due to earthquake events: A case study. Soil Dynamics and Earthquake Engineering; 136, 1-4. http://dx.doi.org/10.1016/j. soildyn.2020.106252

Robinson, T. (2018). Use of scenario ensembles for deriving seismic risk. Proc. Natl. Acad. Sci. Unit. States Am; 115(41), 95329541. https://doi.org/10.1073/ pnas. 1807433115

Rohrmann, B. (1994). Risk perception of different societal groups: Australian findings and crossnational comparison. Aust. J. Psychol; 46, 150-163. https://doi. $\mathrm{rg} / 10.1080 / 00049539408259490$

Sadri, A.M.; Ukkusuri, S.V.; MurrayTuite, P. \& Gladwin, H. (2014). Analysis of hurricane evacuee mode choice behavior. Transport. Res. Part C-Emerg. Technol; 48, 37-46. https://doi.org/10.1016/j. trc.2014.08.008

Santos, R.J., Santos, R.G., Gouzeva, T. \& Velazquez, M.D. (2017). Schoolchildren's earthquake knowledge, preparedness, and risk perception of a seismic-prone 
region of Mexico. Hum. Ecol. Risk Assess; 23(3), 494-507. https://doi. org/10.1080/10807039.2016.118 8368

Shuai, Z., Can, L., Limin, Z., Ming, P., Liangtong, Z. \& Qiang, X. (2020). Quantification of human vulnerability to earthquakeinduced landslides using Bayesian network. Engineering Geology; 265, 1-58. http://dx.doi.org/10.1016/j. enggeo.2019.105436

Sjoberg, L. (1999). Risk perception by the public and by experts: a dilemma in risk management. Hum. Ecol. Rev; 1-9. https://doi. org/10.1027/1016-9040.3.1.1

Tavera, H., Bernal, I. \& Salas, H. (2007). El Sismo de Pisco del 15 de agosto, 2007 (7.9Mw) Departamento de Ica - Perú. Informe Preliminar. Dirección Sismología. Instituto de Geofísica del Perú. Lima, Perú. Consultado 8 de diciembre de 2020. Disponible en: https:// www.eeri.org/wp-content/uploads/ Pisco150807.pdf

UNDESA (2015). World Urbanization Prospects: the 2014 Revision United Nations Department of Economic and Social Affairs Population Division, ST/ESA/ SER.A/352, New York (2015).

Vicente, R., Ferreira, T.M., Maio, R. \& Koch, H. (2014). Awareness, perception and communication of earthquake risk in Portugal: public survey. Procedia Econ. Finan; 18, 271-278. https://doi.org/10.1016/ S2212-5671(14)00940-X

Wyss, M. (2018). Rural populations suffer most in great earthquakes. Seismol. Res. Lett; 89(6), 1991-1997. http:// dx.doi.org/10.1785/0220180236 